\title{
Electromigration-Induced Plasticity: Texture Correlation and Implications for Reliability Assessment
}

\author{
A.S. BUDIMAN, ${ }^{1,2,6}$ P.R. BESSER,${ }^{3}$ C.S. HAU-RIEGE,${ }^{3}$ A. MARATHE ${ }^{3}$ \\ Y.-C. JOO ${ }^{4}$ N. TAMURA, ${ }^{5}$ J.R. PATEL, ${ }^{1,5}$ and W.D. NIX ${ }^{1}$ \\ 1.-Department of Materials Science and Engineering, Stanford University, Stanford, CA 94305, \\ USA. 2.-Spansion Inc., Technology Reliability Engineering (TRE), Sunnyvale, CA 94088, USA. \\ 3.-Advanced Micro Devices, Inc., Sunnyvale, CA, USA. 4.-Department of Materials Science and \\ Engineering, Seoul National University (SNU), Seoul, South Korea. 5.-Advanced Light Source \\ (ALS), Lawrence Berkeley National Laboratory (LBNL), Berkeley, CA 94720, USA. 6.-e-mail: \\ suriadi@stanfordalumni.org
}

\begin{abstract}
Plastic behavior has previously been observed in metallic interconnects undergoing high-current-density electromigration (EM) loading. In this study of $\mathrm{Cu}$ interconnects, using the synchrotron technique of white-beam x-ray microdiffraction, we have further found preliminary evidence of a texture correlation. In lines with strong (111) textures, the extent of plastic deformation is found to be relatively large compared with that of weaker textures. We suggest that this strong (111) texture may lead to an extra path of mass transport in addition to the dominant interface diffusion in $\mathrm{Cu}$ EM. When this extra mass transport begins to affect the overall transport process, the effective diffusivity, $D_{\text {eff }}$, of the EM process is expected to deviate from that of interface diffusion only. This would have fundamental implications. We have some preliminary observations that this might be the case, and report its implications for EM lifetime assessment herein.
\end{abstract}

Key words: Electromigration, copper, interconnects, texture, plasticity, reliability, dislocations, x-ray microdiffraction

\section{INTRODUCTION}

Most interconnect metals are aggregates of crystalline grains. The crystalline lattice of each grain has a characteristic orientation, and a polycrystal is thus characterized by a distribution of orientations-its texture. Texture governs many of the physical, electrical, and mechanical properties of polycrystalline materials. In metallic conductor lines in microelectronics integrated circuits, texture has been known to play important roles in the performance and reliability of the conductors, for instance in electromigration (EM). ${ }^{1}$

Recently, an unexpected mode of plastic deformation has been observed in $\mathrm{Al}^{2,3}$ as well as in $\mathrm{Cu}^{4-6}$ interconnects during EM stressing. This deforma-

(Received June 11, 2008; accepted November 12, 2008; published online December 12, 2008) tion occurs not in any random direction, but always in a certain direction related to the interconnect geometries. Texture and crystal plasticity have a close, intertwined relationship; textures evolve during plastic deformation, while plastic deformation often depends on crystallographic orientations. Understanding crystal plasticity and how it progresses in interconnect lines during EM therefore might lead us to fundamental insights on the role of texture in EM.

The grains that exhibit plastic deformation were always observed to deform (bend, rotate, or polygonize) transverse to the direction of the electron flow in the line, or in other words, across the width of the line. ${ }^{4-6}$ It has been further observed, especially in $\mathrm{Cu}$ lines, ${ }^{4,6}$ that the occurrence of plasticity can be correlated with the availability of a $\langle 112\rangle$ direction in the proximity of the direction of the length of the line (within about $10 \mathrm{deg}$ ). 
In the present study, we investigate a different set of $\mathrm{Cu}$ lines fabricated by a different manufacturer. This set of $\mathrm{Cu}$ lines differs from the previous set $^{4-6}$ in a few ways, chief among them being texture. Using the synchrotron technique of whitebeam x-ray microdiffraction, ${ }^{7}$ developed in Beamline 7.3.3 at the Advanced Light Source, Berkeley $\mathrm{Lab}$, we follow the evolution of plasticity in $\mathrm{Cu}$ polycrystals during similar EM experiments. We observe a strong texture dependence and propose a model to explain the prominent observation.

However, as we establish this texture-plasticity correlation, the effect of plasticity on the $\mathrm{Cu}$ EM degradation process remains unresolved. Based on the results and preliminary observations of the present study, we propose a model suggesting that the effect of plasticity might be that of a damaging one to the Cu EM degradation process. Thus, these results may suggest the more desirable texture of $\mathrm{Cu}$ interconnect lines for EM.

\section{EXPERIMENTAL}

The interconnect test structure used in this study is a variation of a back-end-of-line process for a 65-nm complementary metal-oxide-semiconductor (CMOS) technology, as shown in Fig. 1. In this technology, the dual-damascene $\mathrm{Cu}$ fill process includes a standard Ta-based barrier and $\mathrm{Cu}$ seed, electroplated $\mathrm{Cu}$ fill, post-plating anneal, chemicalmechanical polish, and a dielectric cap layer. Two different interlayer dielectrics (ILD) were integrated with copper: $\mathrm{Cu} /$ low- $k$ ILD (low- $k=$ chemical vapor deposition (CVD) carbon-doped oxide) and $\mathrm{Cu} / \mathrm{hybrid}$ ILD (hybrid $=\mathrm{Cu} /$ low $-k$ at the line level and $\mathrm{Cu}$ /fluorinated tetraethylorthosilicate (FTEOS) at the via layer). Both ILD materials were studied in order to provide a comparison of the extent of plasticity. The metal two (M2) lines were studied after partial removal of the top dielectric to expose the capped $\mathrm{Cu}$ lines (Fig. 1a). In these EM tests, the current was forced from the wide, upper metal layer (M3) into narrow M2 (called a V2M2 test) or from a wide lower metal layer (M1) into a narrow M2 (referred to as a V1M2 test). Both structures are designed to force failure in $\mathrm{M} 2$ at its critical dimension.

The first set of test structures consists of $200-\mu \mathrm{m}$ long lines, approximately $0.2 \mu \mathrm{m}$ thick, and $0.5 \mu \mathrm{m}$ wide. Due to limited beam time, typical of synchrotron experiments, only segments of $50 \mu \mathrm{m}$ length at both cathode and anode ends of the line were studied (Fig. 1a). The dielectric is carbon-based CVD oxide ("low- $k$ " in Fig. 1c). The second set of interconnect test structures was prepared with dimensions similar to those of the first one, but with the hybrid ILD material $\left(\mathrm{SiO}_{2}\right.$-based). The line length is $200 \mu \mathrm{m}$, the thickness is approximately $0.25 \mu \mathrm{m}$, and the width is $0.7 \mu \mathrm{m}$. Similarly, only segments of $50 \mu \mathrm{m}$ length at both cathode and anode ends of the line were studied.

The synchrotron technique of scanning whitebeam x-ray microdiffraction has been described

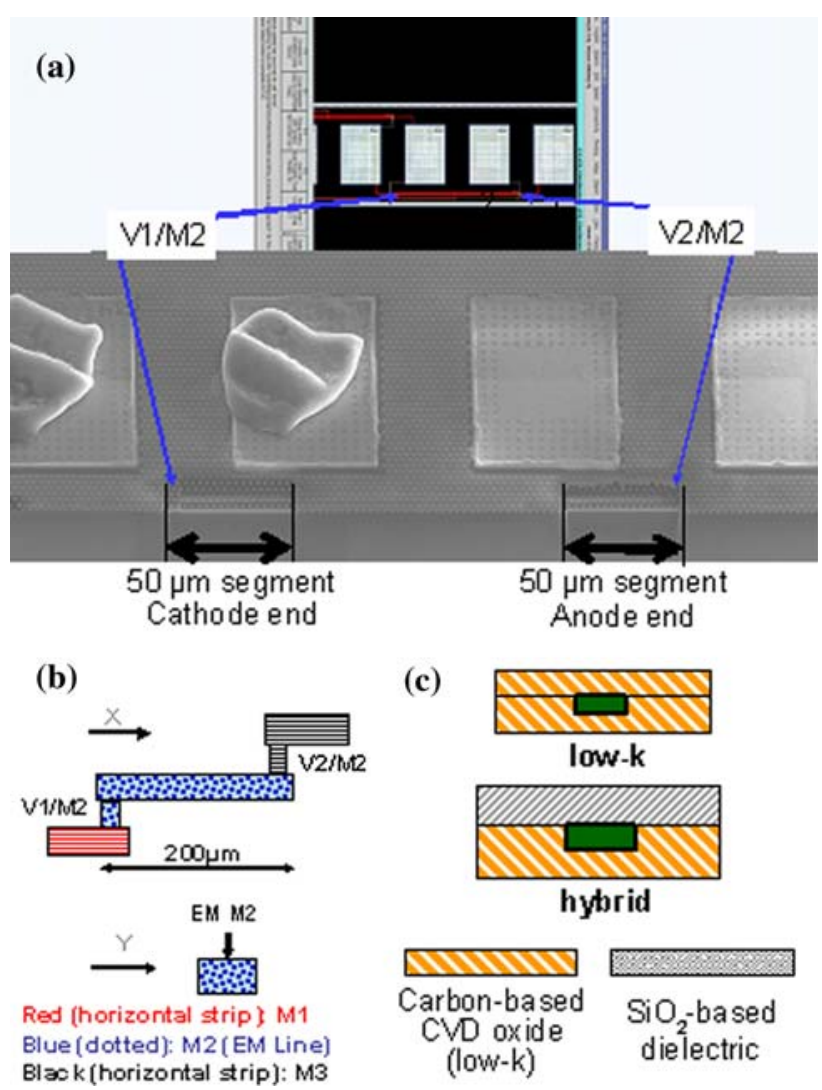

Fig. 1. Scanning electron microscopy (SEM) images and schematic drawings of the $\mathrm{Cu}$ interconnect test structures in this experiment: (a) SEM image of the test structure; (b) in situ EM experiment; (c) two sets of test structures of different dielectric schemes: low- $k$ versus hybrid.

in a complete manner in a previous publication. ${ }^{7}$ The experiment was performed on the Beamline 7.3.3. at the Advanced Light Source, Berkeley, CA. The EM test was conducted at $300^{\circ} \mathrm{C}$ on a viaterminated test structure (Fig. 1b). Current and voltage were monitored at $10-\mathrm{s}$ increments. The sample was scanned by the $0.8 \mu \mathrm{m} \times 0.8 \mu \mathrm{m}$-spotsized white $\mathrm{x}$-ray beam in $0.5-\mu \mathrm{m}$ steps and a Laue pattern was collected at each step using a MAR charge-coupled device (CCD) detector. A complete set of CCD frames takes about $6 \mathrm{~h}$ to $7 \mathrm{~h}$ to collect. The exposure time was $20 \mathrm{~s}$ plus about $6 \mathrm{~s}$ of electronic readout time for each frame. Laue patterns were subsequently analyzed using the x-ray microdiffraction analysis software to obtain information regarding plastic deformation for each crystal grain in the sample. The current was ramped up to $2 \mathrm{~mA}$ $\left(j=2 \mathrm{MA} / \mathrm{cm}^{2}\right)$ and then set at that value for the rest of the test (up to $36 \mathrm{~h}$ ). The ambient temperature in the synchrotron end-station (hutch) where the tests were performed is $20^{\circ} \mathrm{C}$.

\section{RESULTS}

The in situ EM observations will be described first. Figure 2a-c shows the typical evolution of the Laue diffraction spots during the in situ EM test. 


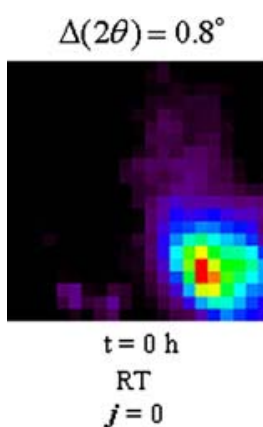

(a)

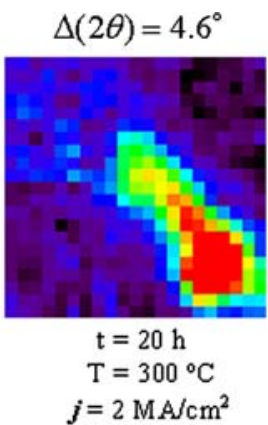

(b)

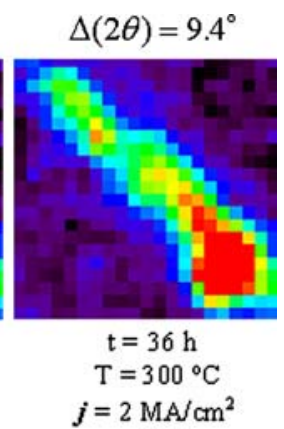

(c)
Fig. 2. The typical evolution of Laue diffractions spots from $\mathrm{Cu}$ interconnect test structures during in situ EM experiments.

Figure 2 is early in the EM test, sampled after $36 \mathrm{~h}$ of testing. The observed broadening of the Laue diffraction spots (streaking) represents plastic deformation of the $\mathrm{Cu}$ grains induced by $\mathrm{EM}^{2-4}$

As the EM test progresses, plasticity is observed in the $\mathrm{Cu}$ grains throughout the line, such as demonstrated in Fig. 3a. Plasticity here may manifest itself either in the form of diffraction spot broadening (streaking) or in the form of diffraction spot splitting (into two or even more different spots). The broadening of the diffraction spots represents crystal bending of the $\mathrm{Cu}$ grains in the line, whereas the split diffraction spots indicate the formation of lowangle boundaries or subgrain structures. ${ }^{2-4}$ From the amount of broadening, the bending of the $\mathrm{Cu}$ crystal can be calculated. From the degree of splitting, the angle of misorientation between subgrains can be determined. ${ }^{2-4}$

Not only was plasticity observed, but also the direction of the plastic deformation is generally consistent across grains throughout the segments of the line under observation, as shown in Fig. 3a. This is consistent with our observation on the previous set of $\mathrm{Cu}$ lines. ${ }^{4-6} \mathrm{Cu}$ grains plastically deform in a direction transverse to the electron flow direction in the line. Such directionality can simply be accommodated by a distribution of same-sign edge dislocations with cores as illustrated in Fig. 3b, i.e., with the $\langle 112\rangle$ line direction of the dislocations all lining up along the direction of electron flow in the line.

Exact grain orientation mapping of these $\mathrm{Cu}$ lines unfortunately could not be obtained in the present study. The x-ray spot size $(0.8 \mu \mathrm{m} \times 0.8 \mu \mathrm{m})$ currently used in ALS beamline 7.3.3 was relatively large for the dimensions of these state-of-the-art interconnect lines. That makes diffraction spot indexation very difficult and thus mapping of grain orientations and other further quantitative analyses unreliable. The few $\mathrm{Cu}$ grains that we show in Fig. 3 were among the limited number of grains in the two $\mathrm{Cu}$ lines for which indexation of the diffraction spots happened to be sufficiently clear and unambiguous for this analysis. In general, the larger the $\mathrm{Cu}$ grains and the more bamboo-like they

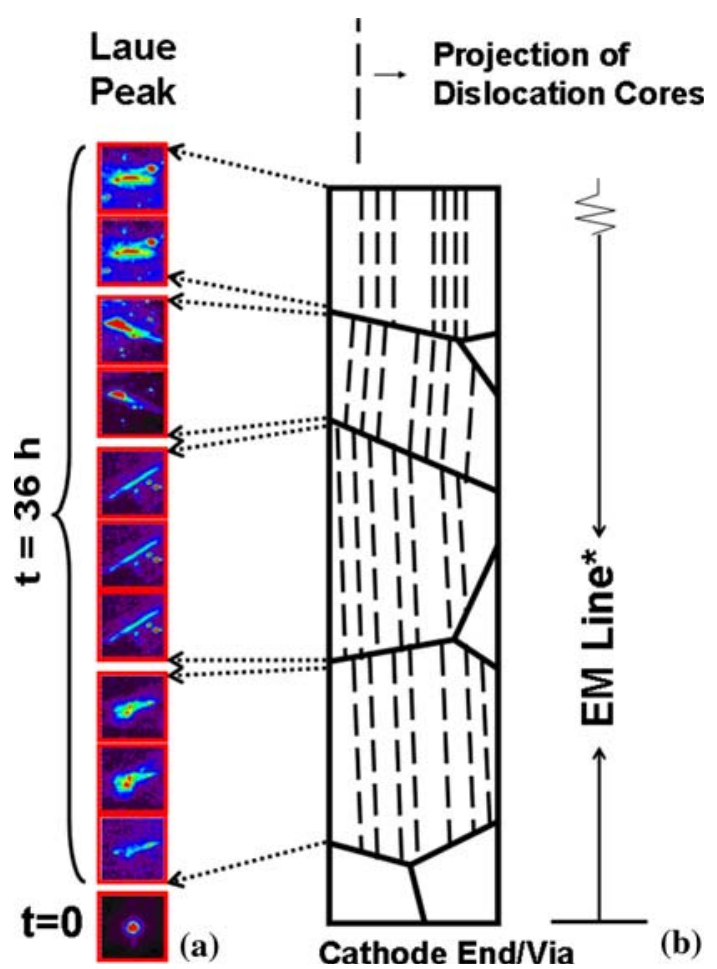

Fig. 3. Laue diffraction images of the cathode end of the line after $36 \mathrm{~h}$ of testing. In the images on the left (a), streaking and/or splitting of $\mathrm{Cu}$ Laue diffractions spots (each image represents a $0.5 \mu \mathrm{m}$ step size) throughout a segment of the line is observed. Dislocations were observed with cores aligned with the direction of the electron flow in the line (consistent with earlier observations ${ }^{4-6}$ ) across grains throughout the length of the segment of the line are observed, as shown schematically on the right (b). ( ${ }^{*}$ Grain map is estimated based on streaking observation; the total line length studied in each cathode or anode end is $50 \mu \mathrm{m}$, and the width is $0.5 \mu \mathrm{m}$ ).

are, the more they diffract sharply and give numerous diffraction spots, thus giving higher confidence in the reliability of these results. That being said, it is fortunate that the evolution of $\mathrm{Cu}$ diffraction spots before and after some period of EM testing can still be compared qualitatively, as demonstrated in Fig. 2.

The extent of the EM-induced plasticity observed in the present samples will now be determined. Figure $4 \mathrm{a}$ and $\mathrm{b}$ shows still different additional diffraction spots observed during this experiment (after EM testing of $36 \mathrm{~h}$, at $300^{\circ} \mathrm{C}$ and $2 \mathrm{MA} / \mathrm{cm}^{2}$ current loading) from $\mathrm{Cu}$ lines with the low- $k$ and the hybrid dielectrics, respectively. The diffraction spots have been converted to $\chi-\theta$ angular space, with $\chi$ running along the direction of the length of the line, and $\theta$ across the direction of the width of the line. The $\chi-\theta$ angles refer to the plane normals responsible for the Laue streaks.

The observed broadening and spot splitting can be used to obtain information about the dislocation structure induced into the grain by EM. For instance, from the streak length of Fig. $4 \mathrm{a}$, as measured in the digital camera image, and knowing the sample-to-detector distance, we determine the 


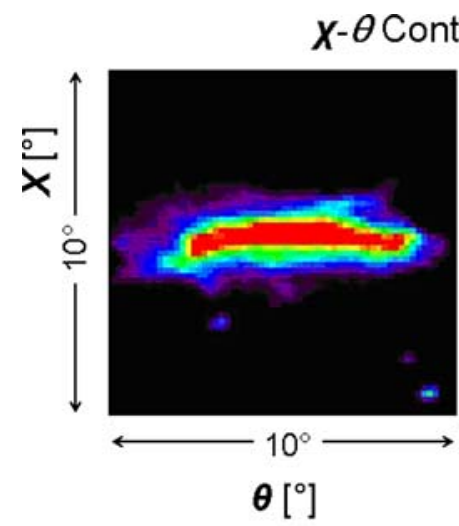

(a)

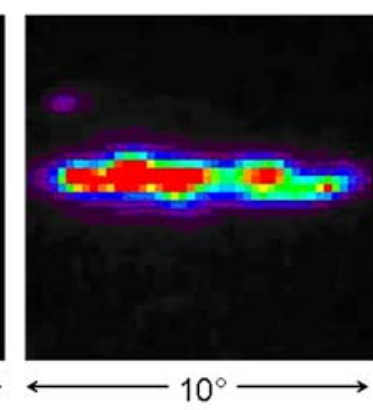

$\theta\left[{ }^{\circ}\right]$

(b)
Fig. 4. Dielectric effects: the Laue peak streaking/splitting observed from $\mathrm{Cu}$ interconnect test structures with (a) low- $k$ and (b) hybrid dielectrics; in $\chi-\theta$ space/contour intensity plot.

curvature angle of the grain to be $9.8 \mathrm{deg}$. Assuming a near-bamboo structure, the grain width is the same as the width of the line $(0.5 \mu \mathrm{m})$, from which we determine the radius of curvature of the grain to be $R=2.34 \mu \mathrm{m}$. The geometrically necessary dislocation (GND) density needed to account for the observed curvature can be calculated from the Cahn-Nye relationship, ${ }^{8,9} \rho=1 / R b$ where $b$ is the Burgers vector. The GND density is then $\rho=1.68 \times$ $10^{15} / \mathrm{m}^{2}$. The total number of dislocations in the area of the cross-section of the $\mathrm{Cu}$ line/grain is approximately 142 .

To obtain quantitative information on polygonization walls (subgrain boundaries) from the spot split in Fig. 4b we observe that the Laue spot splitting, $\Delta_{\theta}=9.1 \mathrm{deg}$. From this misorientation and Burgers' model of a small-angle grain boundary, $\Delta_{\theta}=b / L$, where $L$ is the dislocation spacing, we find that $L=16 \AA$, which amounts to a total of 110 dislocations in the subgrain boundaries in the crosssection of the $\mathrm{Cu}$ line/grain. This translates to a GND density of $\rho=1.12 \times 10^{15} / \mathrm{m}^{2}$.

The extent of the plasticity described here $\left(\rho \sim 10^{15} / \mathrm{m}^{2}\right)$ is observed across all grains throughout the segments of both lines with different dielectric schemes. The significance of the difference in our analysis above, in terms of the extent of the plasticity, as well as its manifestation (grain bending versus polygonization) between the two $\mathrm{Cu}$ lines with different dielectric schemes, requires additional confirmation. Nevertheless they provide a general indication of the extent of plasticity in these $\mathrm{Cu}$ lines.

\section{DISCUSSION}

\section{EM-Induced Plasticity: Texture Correlation}

Compared with the typical observation of the extent of the EM-induced plasticity in the previous set of $\mathrm{Cu}$ interconnect lines, ${ }^{4-6}$ this set of samples exhibits at least a two-order-of-magnitude difference in terms of GND density (Fig. 5). The samples studied previously (referred to as samples A) exhibited $\rho \sim 10^{12} / \mathrm{m}^{2}$ to $10^{13} / \mathrm{m}^{2}$, and the samples in the current study (referred to as samples B) exhibited $\rho \sim 10^{15} / \mathrm{m}^{2}$. Samples A and B differ fairly significantly in dimensions, as well as the dielectric materials used, as shown in Fig. 5 (the dimensions are to scale).

Figure 5 shows the typical evolution of the Laue reflections from the $\mathrm{Cu}$ lines from the initial state $(\mathrm{RT}, j=0, t=$ time $=0$ ) to the EM state (after some $\mathrm{EM}, T=300^{\circ} \mathrm{C}, j \sim 2.0 \mathrm{MA} / \mathrm{cm}^{2}$ to $3.1 \mathrm{MA} / \mathrm{cm}^{2}$, $t \sim 36 \mathrm{~h}$ to $96 \mathrm{~h}$ ). Care was taken in order for the observed intensity contours in the $\chi-2 \theta$ coordinate in Fig. 6 to be comparable (the windows all cover areas of squares of a range of $7 \mathrm{deg}$ to $10 \mathrm{deg}$ in angle space, and the threshold of the lower-bound intensity display was set to be similar). Thus it is obvious from the relative apparent difference in the extent of streaking/splitting of the Laue diffraction spots that the level of plastic deformation that developed during the course of EM in samples B is distinctly larger than that of samples A.

As the two sets of samples (samples A and B) under investigation are provided by different integrated circuit manufacturers, it is not possible to completely quantify the process differences (dielectric type, materials processing, and thermal history) in their technologies in this publication. It is known that the two sets of samples differ in terms of dimension and dielectric materials used; however, it is assumed that the main difference, as far as EM-induced plasticity is concerned, is the crystallographic texture of the $\mathrm{Cu}$ lines. From the texture analysis conducted in our previous study, ${ }^{5}$ we know that samples A have a rather weak (111) texture. As mentioned earlier, the exact grain mapping for the present study (samples B) could not be obtained; however, samples B came from the same manufacturer as the inlaid $\mathrm{Cu}$ lines studied previously. ${ }^{10}$ It is therefore reasonable to assert that samples B would have the typical strong (111) texture as observed by Besser et al. ${ }^{10}$

While other process and dimension differences between these two sets of samples including dimensions and dielectric materials are acknowledged, we believe that these differences cannot satisfactorily explain the differences in the extent of plastic deformation; for example, the $\mathrm{Cu}$ in samples A is surrounded completely by dielectric material, which is a fluorinated $\mathrm{SiO}_{2}$-based dielectric, which is generally believed to constrain the $\mathrm{Cu}$ lines better and should result in less plastic deformation. This is consistent with our observation of samples A versus samples B, but the different dielectric schemes in samples B do not appear to affect the level of plasticity in the $\mathrm{Cu}$ lines. Another example involves the size effect. Wider lines seem to exhibit more plastic deformation in our previous study, ${ }^{4-6}$ such as also shown in Fig. 6a. However, samples B actually are much narrower, and also much smaller 
(a)

\section{Samples A}
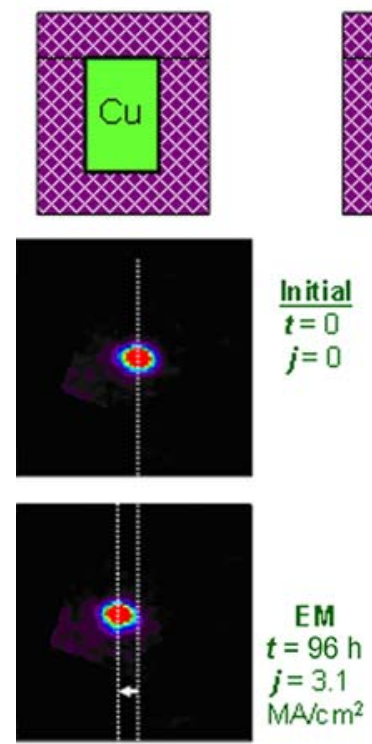

$\rho_{\mathrm{GND}} \sim 10^{12} / \mathrm{m}^{2 *}$
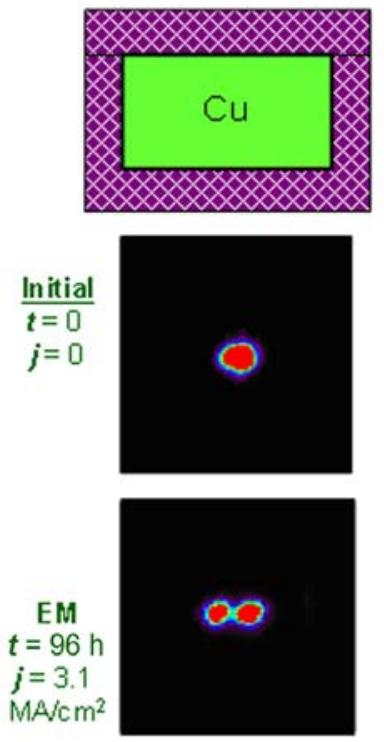

$\rho_{\mathrm{GND}} \sim 10^{13} / \mathrm{m}^{2}$ (b)

\section{Samples B}

[Present Study]
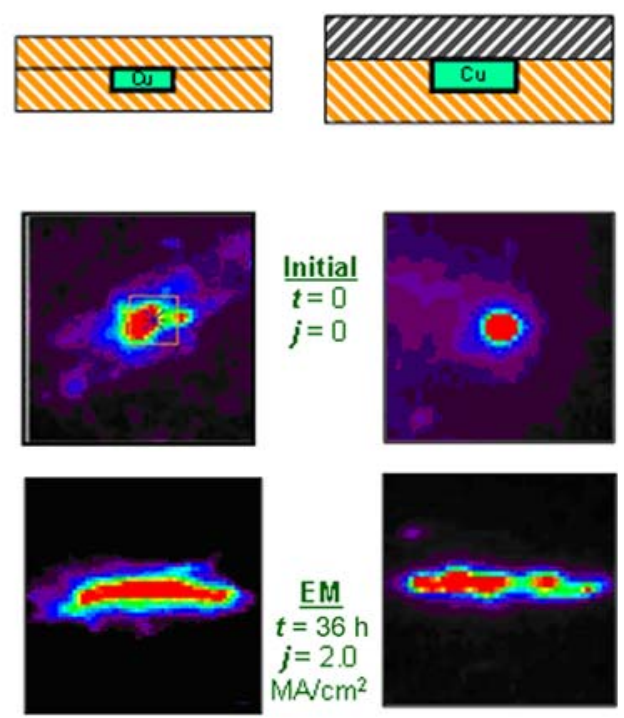

$\rho_{\mathrm{GNO}} \sim 10^{15} / \mathrm{m}^{2}$

Fig. 5. Samples A versus B: the schematic of the cross-sections (color code refers to different materials), typical evolution of Cu Laue diffraction spots (from "Initial" to "EM" states), and lastly the typical densities of GNDs implied by the extent of streaking/splitting of Laue peaks: (a) samples A (Cu lines reported in our previous studies ${ }^{4-6}$ ) and (b) samples B (Cu lines reported herein). They were fabricated by different manufacturers ( ${ }^{\star}$ Taken as that of typical annealed metals).

(a)

$\frac{\text { Samples A }}{\text { [Reference 4-6] }}$

$<111>$

Out-of-plane

orientation

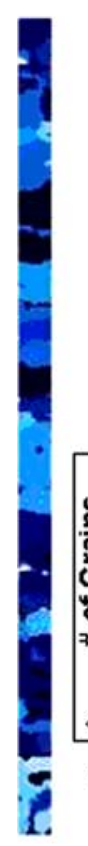

(b)
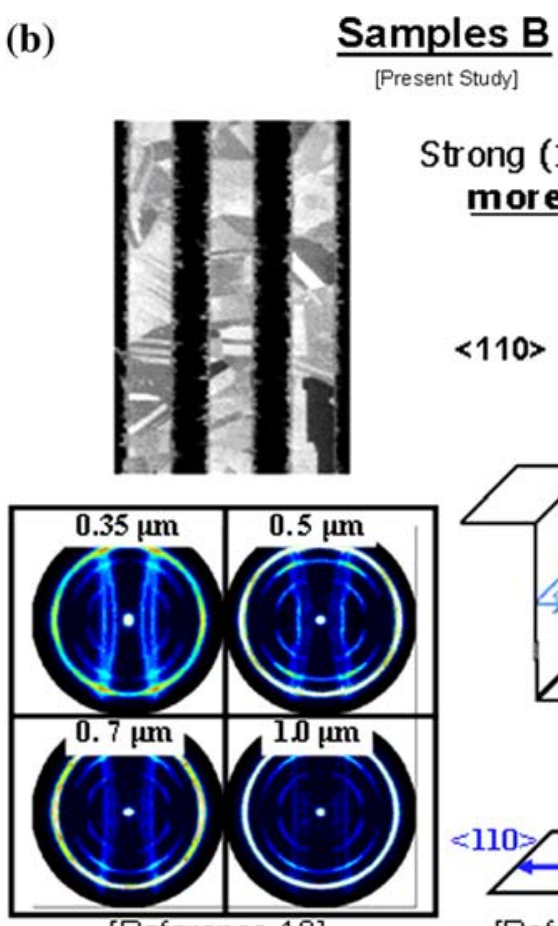

[Reference 10]
Strong (111) texture, more plasticity<smiles>C[Mg][Mg]</smiles>
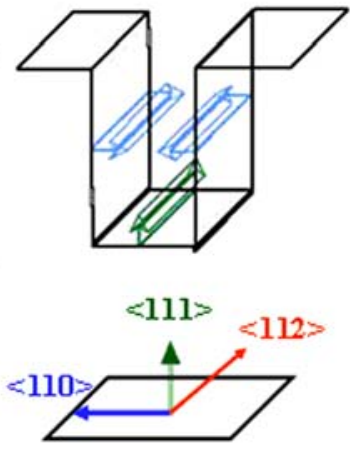

[Reference 10-12]

Fig. 6. Comparison of samples A and B: texture correlation. (a) Samples A (from Refs. 4-6) have a weak (111) texture, and "less plasticity," whereas (b) samples B (Cu lines reported herein) have strong (111) texture, and "more plasticity." The schematic on the right illustrates that the strong preferred in-plane orientation of (111) grains leads to a preferred $\langle 110\rangle$ to the sidewalls and $\langle 112\rangle$ along the direction of length of the lines (courtesy of Besser et al. $^{10}$ ). 
in all cross-sectional dimensions, than samples $\mathrm{A}$, yet samples B exhibit two orders of magnitude more EM-induced plasticity.

Figure 6 is a comparison summary of the known information about the $\mathrm{Cu}$ lines in samples $\mathrm{A}$ versus samples B. First, samples A shows a weak (111) texture, and we found the extent of EM-induced plasticity in the order of $\rho \sim 10^{12} / \mathrm{m}^{2}$ to $10^{13} / \mathrm{m}^{2}$. Subsequently, samples B was found to have $\rho \sim$ $10^{15} / \mathrm{m}^{2}$ after similar EM conditions-a significantly larger amount of EM-induced plasticity. Besser et al. ${ }^{10}$ suggested that samples B have the typical strong (111) texture. This observation of significantly larger EM-induced plasticity in samples B compared with in samples $\mathrm{A}$, is thus consistent with our earlier observation, especially detailed in Ref. 6 , that the occurrence of plastic deformation in a given grain can be strongly correlated with the availability of a $\langle 112\rangle$ direction of the crystal in the proximity of the direction of the electron flow in the line (within an angle of $10 \mathrm{deg}$ ). In $\langle 111\rangle$ out-of-planeoriented grains in a damascene interconnect scheme, the crystal plane facing the sidewall tends to be a $\{110\}$ plane, so as to minimize the interfacial energy. ${ }^{10-12}$ Therefore, it is deterministic rather than probabilistic that the (111) grains will have a $\langle 112\rangle$ direction nearly parallel to the direction of electron flow or the direction of the length of the line. This is illustrated in Fig. 6b.

In samples B, most grains are $\langle 111\rangle$ in outof-plane orientation [such as shown in the focused ion beam (FIB) mapping in Fig. 6b], and thus prefer energetically to have the $\langle 110\rangle$ directions normal to the sidewalls, thus causing a $\langle 112\rangle$ direction to be very close to the direction of the electron flow. When this condition is met, our proposed correlation, discussed in the previous studies, ${ }^{4,5}$ and in greater detail in Ref. 6, suggests that plasticity occurs in these $\mathrm{Cu}$ grains upon $\mathrm{EM}$, and not only did it occur in the present study, but the extent of the plasticity here was rather extreme. Samples A, in the meantime, have only a few grains that are $\langle 111\rangle$ in out-of-plane orientation, which led to the occurrence of plasticity only in these few grains in the $\mathrm{Cu}$ lines after EM. In most other grains (i.e., non- $\langle 111\rangle$ oriented grains), a $\langle 112\rangle$ direction of the $\mathrm{Cu}$ crystal is not likely to be the direction of electron flow of the lines. Thus, plasticity was not observed in many grains in the $\mathrm{Cu}$ lines of samples $\mathrm{A}$.

\section{Dislocation Cores as Fast Diffusion Paths in Metallic Interconnects}

The observed plasticity described above (Fig. 3) leads to a concentration of same-sign edge dislocations with cores running along the direction of electron flow, as illustrated three-dimensionally in Fig. 7. When this configuration of same-sign edge dislocations extends through grains along the full length of the interconnect lines, the dislocation cores can serve as additional paths for diffusion of

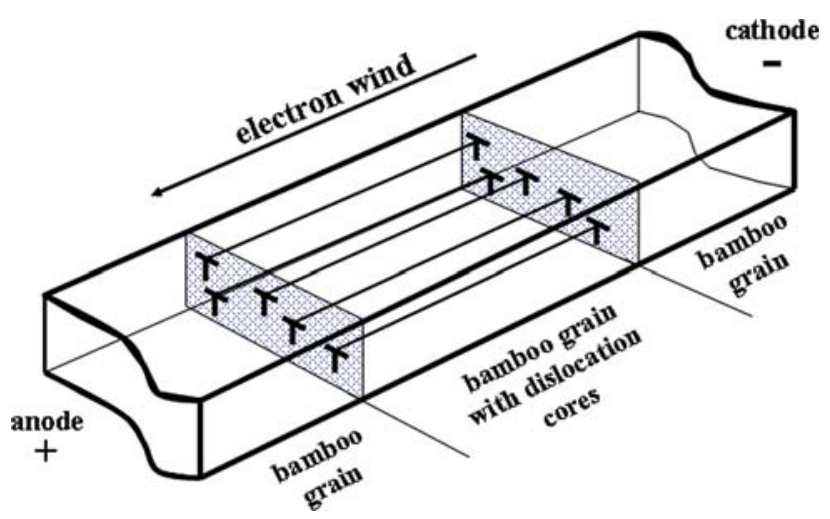

Fig. 7. Schematic of a grain containing same-sign edge dislocations with cores running along the direction of the electron flow in the interconnect line.

atoms from one end of the interconnect line to the other. Dislocation cores are, in general, already recognized as fast diffusion paths, ${ }^{13}$ but in this configuration especially, their contribution to the overall migration of atoms from the cathode to the anode end of the line is even more pronounced. Furthermore, when the concentration of these dislocations becomes high enough, their contribution to the overall effective diffusivity ( $D_{\text {eff }}$ ) can no longer be neglected.

In this context the effective diffusivity can be written as

$$
D_{\text {eff }}=\frac{\delta}{h} D_{\text {int }}+\rho a_{\text {core }} D_{\text {core }}^{\text {eff }}
$$

where $a_{\text {core }}$ is the cross-sectional area of dislocation cores, $D_{\text {core }}^{\text {eff }}$ is the effective core diffusivity, $\rho$ is the dislocation density, and $\delta, h$, and $D_{\text {int }}$ are the effective interface diffusion thickness, the height of the line, and the diffusivity of the interface, respectively. It is necessary here to use $D_{\text {core }}^{\text {eff }}$, the effective core diffusivity (instead of simply $D_{\text {core }}$, the core diffusivity) because, for the dislocation cores to have an effect on mass flow along the full length of the line, a continuous diffusion path (across grains) must be available for atoms to transport from the cathode end to the anode end of the lines. Considering the mostly bamboo grain structure that our interconnect lines have (as shown in Fig. 3), this requires consideration of grain boundary diffusion, as atoms eventually hit the grain boundaries and have to travel some distance in the grain boundary before finding another set of dislocation cores (belonging to the neighboring grain) to continue their travel to the other end of the line. This is illustrated schematically in Fig. 8. Thus, the effective core diffusivity, $D_{\text {core }}^{\text {eff }}$, here will be defined as the effective diffusivity along the dislocation cores when the effect of grain boundary diffusion is taken into account.

It is obvious that only when the effect of grain boundary diffusion is negligible (or in other words, the grain boundary diffusion is a fast enough 


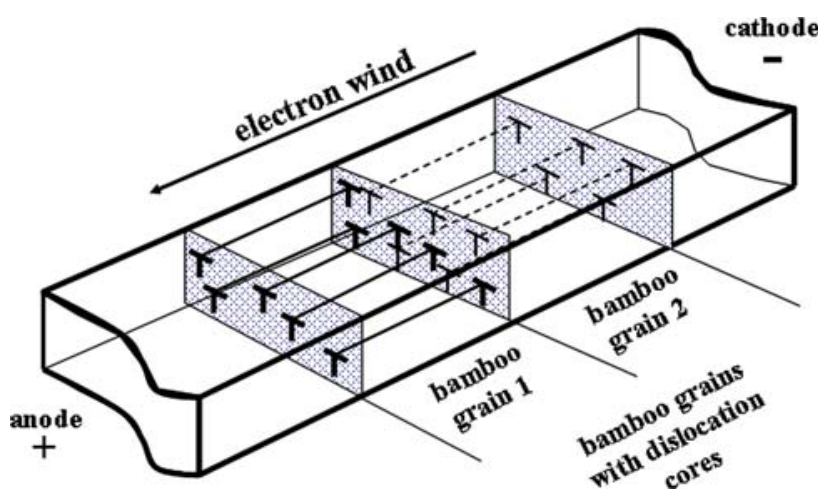

Fig. 8. Illustration of bamboo grains with dislocation cores running along the direction of electron flow in the line under EM bias. Dislocation cores from one grain end at the grain boundaries. Atoms traveling across multiple grains must diffuse along grain boundary regions, before finding another set of dislocation cores in the next grain.

process), can dislocation cores provide a competitive alternative diffusion path and influence the overall effective diffusivity, $D_{\text {eff, }}$ as suggested in Eq. 1 . In order to study quantitatively the impact of this grain boundary diffusion on the overall dislocation core diffusion $\left(D_{\text {core }}^{\text {eff }}\right)$, we derive the kinetics for such a model and arrive at the expression below for $D_{\text {core }}^{\text {eff }}$ (the full derivation is provided in the Appendix),

$$
D_{\text {core }}^{\text {eff }}=D_{\text {core }}\left\{\frac{2 \delta_{\mathrm{gb}} D_{\mathrm{gb}} L}{2 \delta_{\mathrm{gb}} D_{\mathrm{gb}} L+r_{\text {core }}^{2} D_{\text {core }} \ln \left(R / r_{\text {core }}\right)}\right\}
$$

where $\delta_{\mathrm{gb}}$ and $D_{\mathrm{gb}}$ are the effective width and diffusivity of the grain boundary, respectively, $L$ is the overall length of the diffusion path, $r_{\text {core }}$ is the radius of the dislocation core, and $R$ is the mean distance in the grain boundary to the next dislocation core.

The influence of grain boundary diffusion on the overall/effective dislocation core diffusivity, $D_{\text {core }}^{\text {eff }}$, thus depends on the relative magnitude of the two terms in the denominator in Eq. 2. If

$$
2 \delta_{\mathrm{gb}} D_{\mathrm{gb}} L \gg r_{\text {core }}^{2} D_{\text {core }} \ln \left(R / r_{\text {core }}\right),
$$

then, as is evident from Eq. 2, $D_{\text {core }}^{\text {eff }}$ degenerates into simply $D_{\text {core }}$, or in other words, there is very little influence of the grain boundary diffusion in the overall scheme in Fig. 8. If the reverse is true, $D_{\text {core }}^{\text {eff }}$ will be much smaller than $D_{\text {core }}$, in which case it is clear that the grain boundary slows down the overall diffusion significantly.

Referring now to the textbook values for grain boundary and core diffusion compiled in Table I, ${ }^{14-17}$ and compared in Table II, it is evident that the $2 \delta_{\mathrm{gb}} D_{\mathrm{gb}} L$ term in Eq. 2, as well as in Eq. 3, is at least four orders of magnitudes larger than the $r_{\text {core }}^{2} D_{\text {core }} \ln \left(R / r_{\text {core }}\right)$ term. This leads to the degeneration of $D_{\text {core }}^{\text {eff }}$ into simply $D_{\text {core }}$ in Eq. 2 , reducing to Eq. 4

$$
D_{\text {core }}^{\text {eff }} \approx D_{\text {core }}
$$

The practical implication of Eq. 4 is that a practically continuous pipe (dislocation core) diffusion path across multiple grains between the cathode end and the anode end of the line is indeed available for atomic transport in the $\mathrm{Cu}$ test structures under accelerated EM testing.

An extreme would be to take the activation energy for grain boundary diffusion, $E_{\mathrm{A}, \mathrm{gb}}$, to be the $E_{\mathrm{A}}$ for lattice diffusion, which is $2.04 \mathrm{eV}^{14}$ This is a much higher activation energy than that of grain boundary diffusion. In this case, we show that the combined diffusivity would be dominated by such slow diffusion in the hypothetical grain boundary. The effective transport through dislocation cores in this case would be slowed down by nearly

Table II. Values of the Two Parameters/Terms in Eq. 3 (or the Denominator of Eq. 2) Calculated Based on Values Listed in Table I

\begin{tabular}{lc}
\hline Parameter/Term & Value \\
$2 \delta_{\mathrm{gb}} D_{\text {gb }} L$ & $3.2 \times 10^{-30} \mathrm{~m}^{4} / \mathrm{s}$ \\
$r_{\text {core }}^{2} D_{\text {core }} \ln \left(R / r_{\text {core }}\right)$ & $5.0 \times 10^{-35} \mathrm{~m}^{4} / \mathrm{s}$ \\
\hline
\end{tabular}

Table I. Values Used to Determine the Influence of Grain Boundary Diffusion on the Overall Transport Kinetics in the Cu Line under EM. The Diffusivities $\left(D_{\text {gb }}, D_{\text {core }}\right)$ are Described in the Usual Way by $D=D_{0}$

\begin{tabular}{|c|c|c|}
\hline Variable & Value & Reference/Remarks \\
\hline $\begin{array}{l}T \\
E_{\mathrm{A}, \mathrm{gb}} \\
\delta_{\mathrm{gb}} D_{\mathrm{gb}} \\
L \\
\gamma_{\text {core }} \\
E_{\mathrm{A}, \text { core }} \\
r_{\text {core }}^{2} D_{\text {core }} \\
\rho_{\mathrm{GND}} \\
R\end{array}$ & $\begin{array}{c}300^{\circ} \mathrm{C}=573 \mathrm{~K} \\
1.08 \mathrm{eV} \\
1.6 \times 10^{-24} \mathrm{~m}^{3} / \mathrm{s} \\
1 \mu \mathrm{m} \\
0.25 \AA \\
0.21 \mathrm{eV} \\
7.3 \times 10^{-36} \mathrm{~m}^{4} / \mathrm{s} \\
10^{15} / \mathrm{m}^{2} \\
22 \mathrm{~nm}\end{array}$ & $\begin{array}{c}\text { Following } T_{\text {test }} \text { in section "Experimental" } \\
\text { Refs. } 14-17 \\
\text { Calculated, Ref. } 14 \\
\text { Estimated based on section "Results" } \\
\text { Ref. } 14 \\
\text { Ref. } 14 \\
\text { Calculated, Ref. } 14 \\
\text { As observed in section "Results" } \\
R=1 / 2 \rho_{\text {core }}\end{array}$ \\
\hline
\end{tabular}
$\exp \left(-E_{\mathrm{A}} / k T\right)$, Where $E_{\mathrm{A}}$ is the Activation Energy, $D_{\mathrm{o}}$ is the Pre-exponential Constant, and $k$ is Boltzmann's Constant. The Subscript “gb” Refers to Grain Boundary Diffusion 


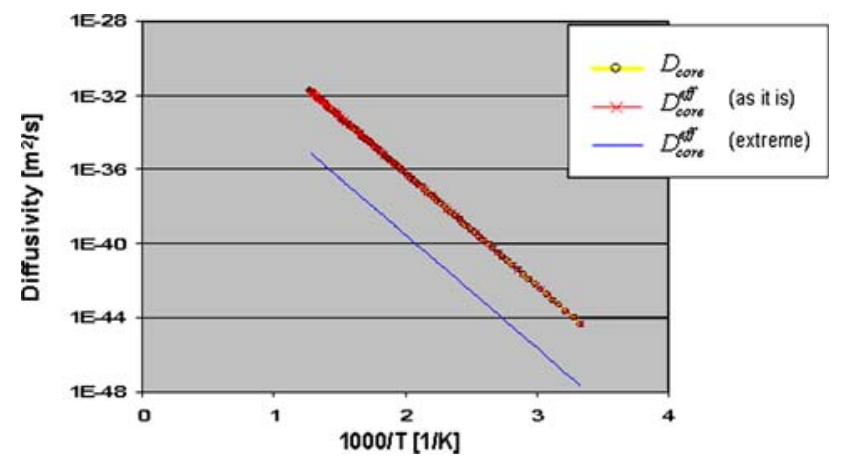

Fig. 9. Comparison of diffusivities as a function of temperature between $D_{\text {core }}$ (only dislocation core diffusion, no grain boundary), $D_{\text {core }}^{\text {eff }}$ (considering the effect of grain boundary; as it is-as shown in Table II), and an extreme $D_{\text {core }}^{\text {eff }}$ (considering the effect of grain boundary diffusion as if it is lattice diffusion). Diffusivities were calculated using values summarized in Table I.

four orders of magnitude due to the effect of the hypothetical grain boundary. This is shown in Fig. 9, which compares calculated diffusivities as a function of temperature for three different schemes of diffusion ( $D_{\text {core }}, D_{\text {core }}^{\text {eff }}$ "as it is," and $D_{\text {core }}^{\text {eff }}$ "extreme," as defined above). The $D_{\text {core }}^{\text {eff }}$ "extreme" line is close to four orders of magnitude lower than the $D_{\text {core }}^{\text {eff }}$ line (crosses) and the $D_{\text {core }}$ line (open circles), which are practically on top of each other, as suggested by Eq. 4 .

It is therefore reasonable to propose that a fully continuous network of dislocation cores running along the direction of the length of the line, slowed only by less than $0.01 \%$ by grain boundary diffusion, exists in the $\mathrm{Cu}$ interconnect lines studied during EM under accelerated test conditions in this study. This makes it a viable alternative for global transport of atoms in $\mathrm{Cu}$ interconnects under EM bias.

The existence of a viable path of dislocation core diffusion alone, however, is not sufficient to influence the overall kinetics in Eq. 1, i.e., if the dislocation density ( $\rho$ or $\rho_{\mathrm{GND}}$ ) is not high enough. We cover this situation below and show the importance of the experimental results of the present study in understanding the overall kinetics in $\mathrm{Cu}$ interconnect lines under EM.

\section{Density of Core Dislocations ( $\left.\rho_{\text {core }}\right)$ : Extent of Plasticity}

Diffusion along dislocation cores (pipe diffusion) has been commonly included in models of diffusioncontrolled deformation in bulk materials. ${ }^{14} \mathrm{Suo}^{18}$ considered the motion and multiplication of dislocations under the influence of an electric current in a conductor line, and suggested that EM-driven dislocation multiplication could itself lead to dislocation densities high enough to affect EM degradation processes. Oates, ${ }^{19}$ however, did not see any diffusivity effects that could be attributed to dislocations in his experimental study. Baker et al. ${ }^{13}$ through their experimental study of nanoindented

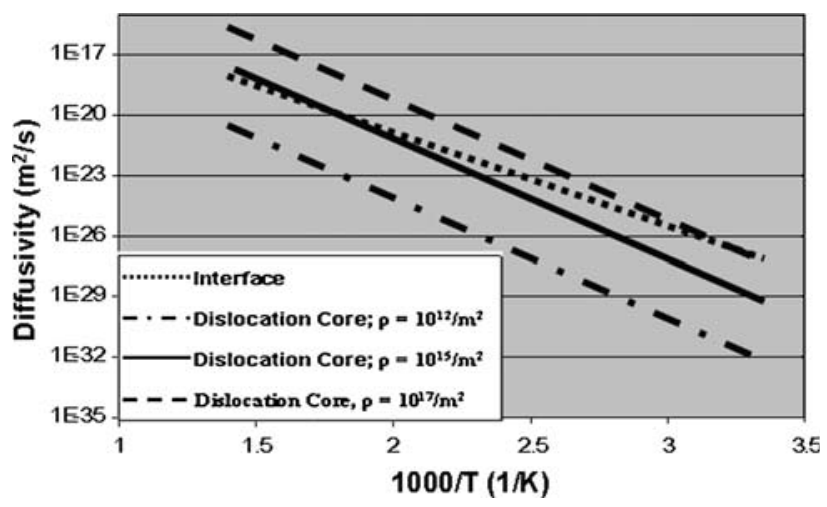

Fig. 10. Calculated diffusivities as a function of temperature between the interface diffusion path and those of dislocation cores of various densities in $\mathrm{Cu}$ interconnect lines $\left(10^{12} / \mathrm{m}^{2}, 10^{15} / \mathrm{m}^{2}\right.$, and $10^{17} / \mathrm{m}^{2}$ ). Each diffusion mechanism is assumed to act alone.

$\mathrm{Al}$ lines (width $=1 \mu \mathrm{m}$, mean grain size $=1.1 \mu \mathrm{m}$ ) showed that the effect of a dislocation density of $10^{16} / \mathrm{m}^{2}$ is comparable to diffusion through a grain boundary. These studies all essentially suggest that, if the dislocation density is sufficiently high, it may affect the overall EM degradation processes in metallic interconnects, and thus could have fundamental implications.

We have, earlier in the manuscript, established that dislocations with cores running along the electron flow direction and densities on the order of $10^{15} / \mathrm{m}^{2}$ are present in the $\mathrm{Cu}$ lines undergoing $\mathrm{EM}$ (accelerated test conditions) for $36 \mathrm{~h}$. Figure 10 shows a comparison of calculated diffusivities as a function of temperature between the interface diffusion path and those of dislocation cores of various densities in $\mathrm{Cu}$ interconnect lines $\left(10^{12} / \mathrm{m}^{2}, 10^{15} / \mathrm{m}^{2}\right.$, and $10^{17} / \mathrm{m}^{2}$ ) when each diffusion mechanism is assumed to act alone. The diffusivities are calculated based on diffusion coefficient values in the literature ${ }^{14,15}$ for $\mathrm{Cu}$ interconnect lines (tabulated in Table III), and for the interconnect dimensions as used in the present study.

Table III. Values Used to Determine Diffusions in Cu Interconnects as a Function of Temperature (Fig. 10). $D_{\mathrm{o}}$ is the Pre-exponential Constant and $E_{\mathrm{A}}$ is the Activation Energy. The Subscripts "int" and "core" Refer to Interface and Core Diffusion, Respectively. $\delta$ is the Effective Interface Diffusion Thickness, $h$ is the Thickness of the Cu Lines, and $a_{\text {core }}$ is the Area of a Dislocation Core

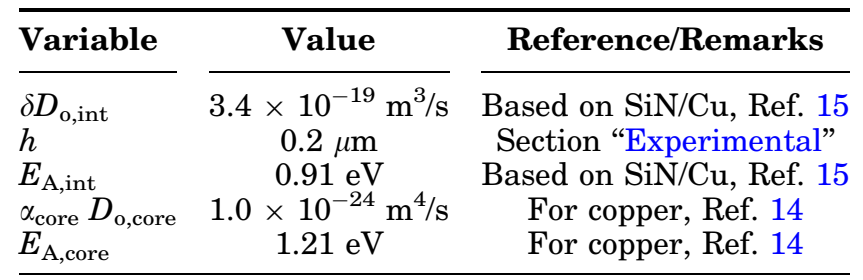


The dislocation density observed in the present study $\left(\rho_{\text {GND }}=\rho_{\text {core }}=10^{15} / \mathrm{m}^{2}\right)$ is illustrated as the solid line in Fig. 10, illustrating that dislocation core diffusion is on the same order of magnitude as that of interface diffusion (the dotted line) at the test conditions $\left(T=300^{\circ} \mathrm{C}\right.$ or $\left.1,000 / T=1.75 / \mathrm{K}\right)$. Thus, a core dislocation density of $10^{15} / \mathrm{m}^{2}$ is the dislocation density threshold necessary for dislocation core diffusion to be on a par with interface diffusion. In other words, at this dislocation density the contribution of dislocation cores to the overall/ effective diffusivity in the $\mathrm{Cu}$ line during accelerated EM is expected to be at least of the same order of magnitude as interface diffusion and thus cannot be neglected.

It is to be noted, however, that at temperatures at or below $100^{\circ} \mathrm{C}$, the required dislocation density for cores to have a significant contribution to the diffusion would be on the order of $10^{17} / \mathrm{m}^{2}$ (the dashed line in Fig. 10). These lower temperatures correlate with the typical use or operational conditions of the interconnects. The typical initial (as-fabricated) dislocation density in $\mathrm{Cu} /$ metallic lines was taken to be $10^{12} / \mathrm{m}^{2}$ (following Ref. 13), and the corresponding diffusivity is shown by the dashed-dotted line in Fig. 10.

It is therefore reasonable to propose that the contribution from the dislocation core diffusion (the second term in Eq. 1) can no longer be neglected in the $\mathrm{Cu}$ lines now that we have evidence of the existence of such high density of dislocation cores in the real $\mathrm{Cu}$ interconnect structure. It is certainly true in the $\mathrm{Cu}$ lines investigated in the present study especially during EM at accelerated test conditions. The contribution from dislocation cores would enhance the EM diffusion, or in other words, the total EM flux $\left(J_{\mathrm{EM}}\right)$, since the total or overall diffusion includes the existing, usually dominant, interface diffusion plus the observed dislocation core (or pipe) diffusion. The increase in this core diffusion to the point of significance in the overall EM diffusion is related to the kind and the extent of plasticity induced by the EM process itself (i.e., through the increase in the core dislocation density from the pre-EM density $\rho_{\text {core }}=10^{12} / \mathrm{m}^{2}$ to the observed density $\rho_{\text {core }}=10^{15} / \mathrm{m}^{2}$ ).

The result of this study gave a key piece of experimental evidence that opens up the possibility that such a high dislocation density may generally be present in $\mathrm{Cu}$ test structures undergoing EM. The circumstances and the important implications of this special configuration of dislocation cores for the EM degradation processes warrant discussion, as do the EM reliability assessment methodologies. With $\rho_{\mathrm{GND}} \sim 10^{15} / \mathrm{m}^{2}$ observed in this study, and $D_{\text {core }}^{\text {eff }}$ that is not much reduced by grain boundary diffusion (as derived in the Appendix), the second term in Eq. 1 (i.e., the contribution of the dislocation core diffusion) can indeed no longer be neglected. This means it will have important implications for fundamental understanding of the EM degradation processes, as well as EM reliability assessment methodologies.

\section{The Extra Dependency on $j$ : Implications for EM and Reliability Assessment Methodology}

If $\rho$ should increase with $j$, then we will find that $D_{\text {eff }}$ (the overall/effective diffusivity of the EM process) should also increase with $j$. Consequently, there will be an extra EM flux, and thus an extra reduction in the mean time to failure (MTF) of the device with increasing $j$. This is an extra dependency on $j$, which would manifest itself in the value of the current density exponent, $n$ (in Black's equation ${ }^{20}$ ), being $>1$.

$$
\mathrm{MTF}=A\left(\frac{1}{j}\right)^{n} \exp \left(\frac{E_{A}}{k T}\right)
$$

The fact that $n$ is usually found in real cases to be $>1$ (as opposed to $n=1$ for the prevailing model of void growth limited failure ${ }^{21-23}$ ) suggests that this extra dependency on $j$, especially under hightemperature test conditions, could be due to dislocation core diffusion. In other words, this higher $n$ could be traced back to the higher level of plasticity in the crystal and, the closer $n$ is to unity, the less plasticity must have influenced the EM degradation process.

Kirchheim and Kaeber ${ }^{24}$ experimentally observed the MTF dependency on current density, $j$, in an Al conductor line, for a wide range of $j$, as shown in Fig. 11 (the solid black dots with error bars are the original data points). This clearly shows that, at low current densities, the MTF data is best fit by $n=1$ (straight solid line), while at higher current densities the MTF data is better fit by $n>1$ (curved dotted line). Kirchheim and Kaeber however suggested in their paper that these deviations occurring

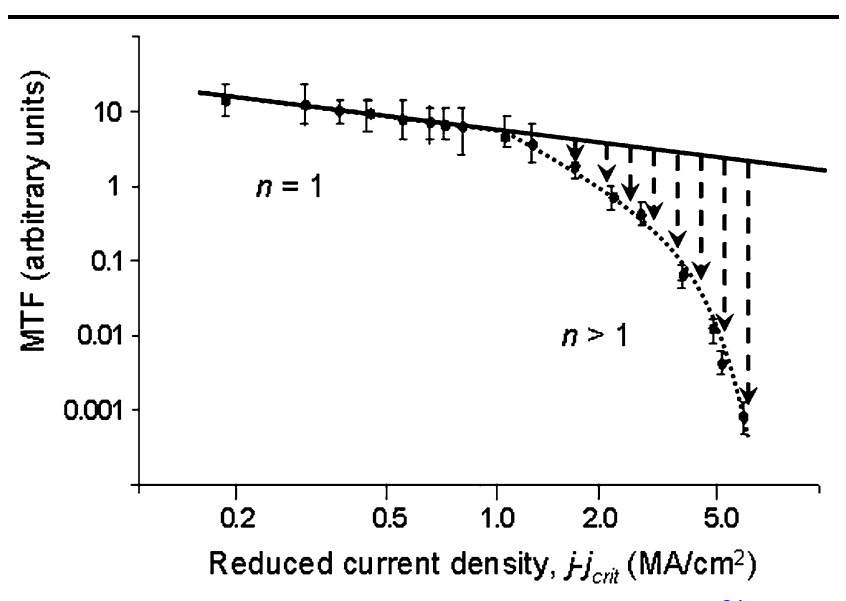

Fig. 11. Kirchheim and Kaeber's experimental MTF data ${ }^{24}$ (reproduced manually here to the highest accuracy possible from Ref. 24; for clarity and improved image resolution) as a function of reduced current density, $j-j_{\text {crit }}$ (all the solid features). The dotted and dashed lines are added to lead to our argument. 
at higher current densities might have been caused by Joule heating. ${ }^{24}$

Plasticity, especially in the form described in this paper as well as in the literature,${ }^{2-6,25,26}$ could just as likely be the source of such deviations of MTF dependency on $j$ at high current densities. As $j$ increases, plasticity also increases, leading to increasingly higher EM fluxes (dashed arrowed lines in Fig. 11), thus increasingly lower MTF, and therefore eventually a current density exponent $n>1$ has to be used to fit the failure time distribution.

However, under use conditions in which the temperature is much lower (e.g., $100^{\circ} \mathrm{C}$ ), the level of $\rho$ associated with such elevated diffusivity is almost impossible to reach, so that this plasticity-amplified diffusivity is associated only with the high temperature and high current density of the accelerated EM test. In other words, there is not likely to be much plasticity under use conditions, and thus the diffusivity is dominated only by interface diffusion, and consequently the MTF dependency on $j$ should follow the $n=1$ line. This is consistent with the observations of Kirchheim and Kaeber ${ }^{24}$ (Fig. 11 shows data following the $n=1$ line at low reduced current densities of $0.2 \mathrm{MA} / \mathrm{cm}^{2}$ to $1 \mathrm{MA} / \mathrm{cm}^{2}$ ). This interpretation of the Kirchheim and Kaeber data is consistent with the physical model (void growth limited failure) which has also been observed through in situ EM studies by Zschech et al. ${ }^{27}$ on samples/materials similar to those used in the present study.

It can be further stated that plasticity-amplified diffusivity is simply an extra mode of deformation under test conditions (which is not typically present under use conditions), and that its effect is wholly captured in the $n$ value being greater than unity. This plasticity-inflated $n$ could thus lead to inaccurate extrapolations of lifetimes under use conditions. This is illustrated in Fig. 12.

Figure 12 illustrates the danger of overestimating device lifetime if, for example, we simply take the three MTF data points under accelerated $j$ (the three solid black triangles in Fig. 12), and based on these data points, we calculate $n$ (which will be larger than 1), and then we use this $n$ to extrapolate from the accelerated condition (high $j$ ) to the use condition (low $j$ ). That extrapolation is shown by the dashed-dotted line in Fig. 12, and is clearly an overestimation of the device's actual lifetime (approximated by the MTF data at low reduced current density).

To improve the accuracy of the reliability assessment of devices under use conditions, we propose that the effect of plasticity has to be removed from the EM lifetime equation. This can be done simply by insisting on $n=1$ in our lifetime assessment (i.e., $j_{\max }$ calculation) which in most typical EM test conditions will result in a more conservative prediction of device lifetime, such as that illustrated by the dashed line in Fig. 12.

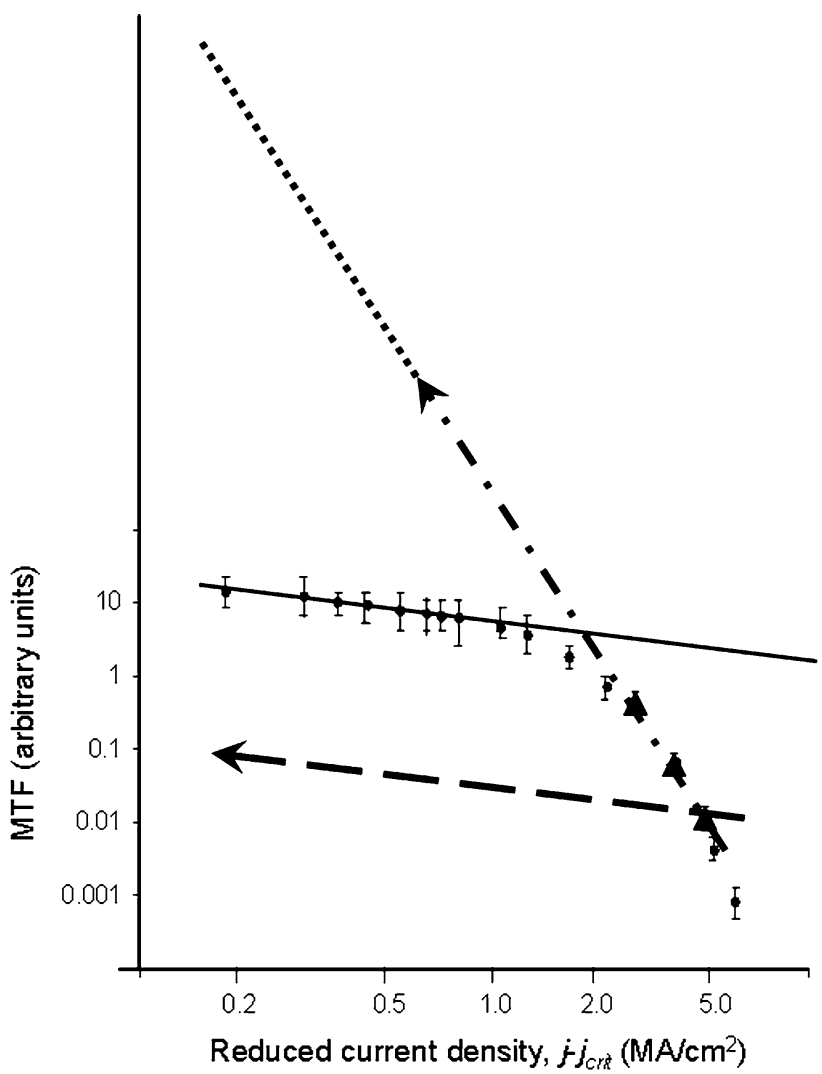

Fig. 12. Illustration of the impact of the current exponent $(n)$ on the extrapolated lifetime. The danger of overestimation of device lifetime by using $n>1$ is shown (dotted-dashed line), as is the more conservative extrapolation using $n=1$ (dashed line), which is closer to the actual device lifetime in use conditions. The reproduced Kirchheim and Kaeber's experimental MTF data ${ }^{24}$ is again used here for illustrative purposes.

\section{CONCLUSIONS}

Plastic deformation behavior of $\mathrm{Cu}$ polycrystals during EM experiments has been observed using a synchrotron technique involving white-beam x-ray microdiffraction. The occurrence of the plastic behavior observed in these $\mathrm{Cu}$ lines appears to depend on the availability of a $\langle 112\rangle$ direction along the length of the $\mathrm{Cu}$ lines as the rotation axis of the plastic deformation. Furthermore, with the present set of $\mathrm{Cu}$ lines, we found that the extent of the EMinduced plasticity was significantly larger compared with that observed in a previous study. We propose that the crystallographic texture of the $\mathrm{Cu}$ lines plays a primary role in controlling the plastic behavior of the interconnect lines. Strong (111) texture leads to high preference of $\langle 112\rangle$ direction along the length of the line, and this subsequently leads to a higher tendency for the grains to behave plastically in response to EM stressing. The extent and configuration of dislocations in the $\mathrm{Cu}$ grains induced during this accelerated EM testing could lead to another competing EM diffusion mechanism in addition to interface diffusion. It is suggested that this plasticity effect can be correlated to the 
measured value of current density exponent, $n$, in Black's equation and thus has important implications for the way device lifetime/reliability is assessed. A new method of lifetime extrapolation for EM by insisting on the use of $n=1$ is proposed, which avoids potentially dangerous and overestimated lifetime prediction based on current methodologies using the measured value of $n$ from accelerated test conditions.

\section{ACKNOWLEDGEMENTS}

The authors would like to thank Advanced Micro Devices (AMD) for generous support and for fabricating the samples in the study. Bryan Tracy of Spansion provided the FIB images in Fig. 1. One of the authors (A.S.B.) would like to thank John M. Ennals, the the AMD/SRC Program Manager at AMD, for the opportunity of a Summer Internship Program in 2006. Both A.S.B. and W.D.N. gratefully acknowledge support by the US Department of Energy, Office of Basic Energy Sciences through Grant No. DE-FG02-04ER46163. The Advanced Light Source (ALS) is supported by the Director, Office of Science, Office of Basic Energy Sciences, of the US Department of Energy under Contract No. DE-AC02-05CH11231 at the Ernest Orlando Lawrence Berkeley National Laboratory (LBNL).

\section{APPENDIX}

The parameter $D_{\text {core }}$ takes into consideration only diffusion along the dislocation cores for the overall length of specimen of interest, which would be true only for a single crystal $\mathrm{Cu}$ along the full length of the line. In this study, the lines are polycrystalline, and thus the effect of grain boundary diffusion must be considered. Here we study quantitatively the impact of grain boundary diffusivity on the overall dislocation core diffusion $\left(D_{\text {core }}^{\text {eff }}\right)$.
We first consider the grain boundary region as illustrated in Fig. 13, and suggest a relation between the mean distance, $R$, that atoms need to travel in the grain boundary region before finding another set of dislocation cores belonging to the next grain, and the dislocation density, $\rho_{\text {core }}$, in the $\mathrm{Cu}$ lines induced by the EM process itself. As can be expected, $R$ is inversely related to $\rho_{\text {core }}$ (or in other words, the more dislocation cores in the cross-section of the $\mathrm{Cu}$ lines, the smaller the diffusion distance in the grain boundary region).

We can deduce, based on the illustration in Fig. 13, that

$$
R=\frac{l}{\sqrt{2}} ; \quad \rho_{\text {core }}=\frac{1}{l^{2}}
$$

and thus

$$
R=\frac{1}{\sqrt{2 \rho_{\text {core }}}},
$$

where $l$ is the mean distance between dislocation cores, and $R$ is the mean distance of grain boundary diffusion. We can now consider the effective diffusion along a hypothetically continuous dislocation core, as well as the actual diffusion along the dislocation core and along the grain boundaries connecting dislocation cores in one grain with those in another, for a grain size, $L$. For the effective core diffusion along the hypothetical dislocation core of length $L$, the flux, $J_{\text {eff, }}$ can be expressed as

$$
J_{\text {eff }}=-\frac{D_{\text {core }}^{\text {eff }} c}{k T} \frac{\mathrm{d} \mu}{\mathrm{d} x}
$$

where $c=1 / \Omega(c=$ concentration of diffusing species; $\Omega=$ atomic volume), $\mu$ is the chemical potential, $x$ is the axis of the diffusion direction along the dislocation core, and $D_{\text {core }}^{\text {eff }}, k$ and $T$ have been defined before. This is illustrated in Fig. 14a.
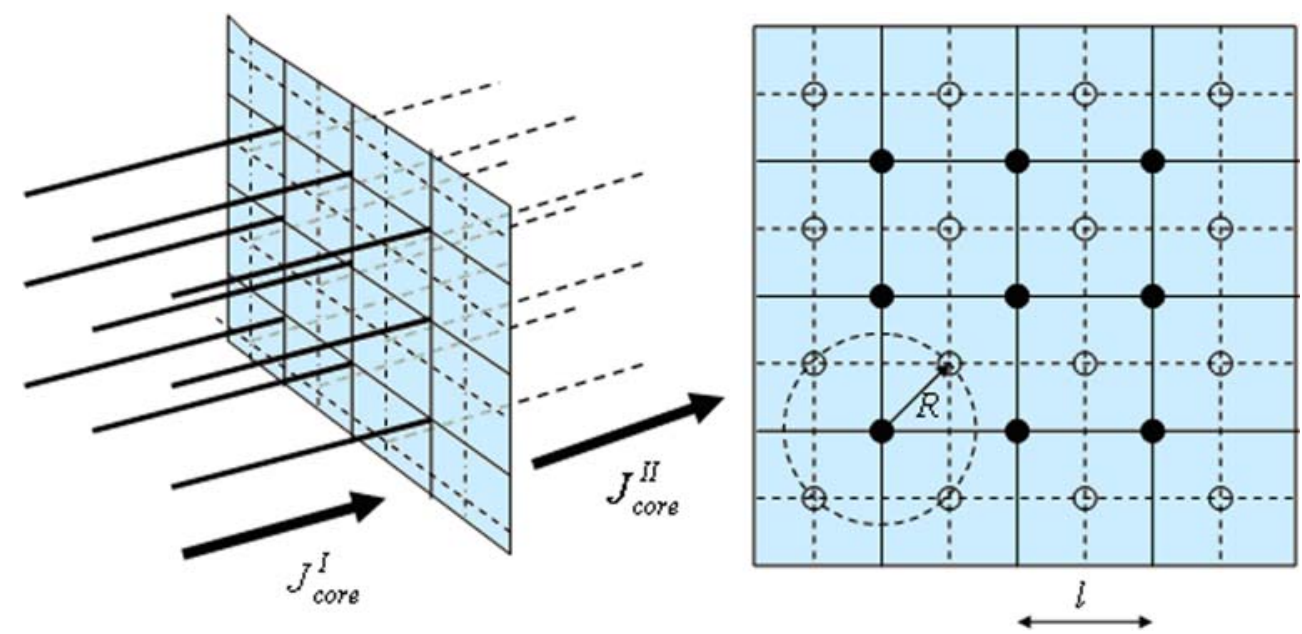

Fig. 13. Illustration of the grain boundary region of two bamboo grains with dislocation cores running along the direction of the electron flow in the line under an EM bias. Atoms traveling along the cores of the first grain (solid lines, closed circles) must diffuse in grain boundary regions for the distance $R$ before finding another set of dislocation cores in the next grains (dashed lines, open circles). 
(a)

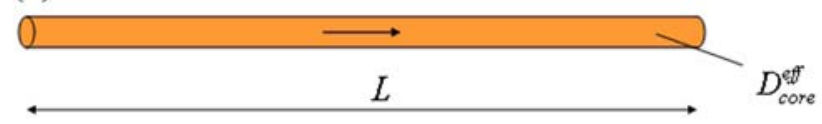

(b)

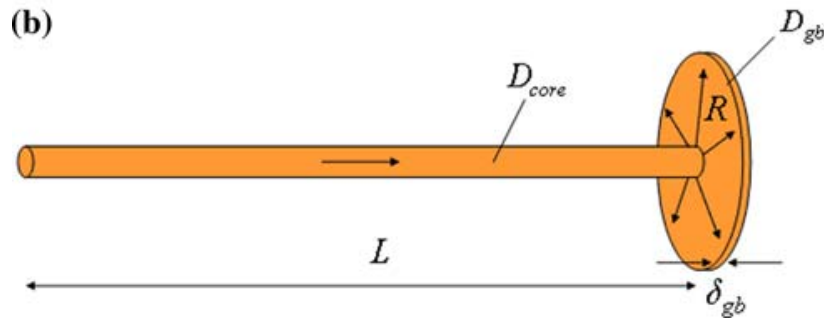

Fig. 14. Illustration of (a) hypothetically continuous dislocation core diffusion along a specified diffusion distance, $L$, and (b) the combined effects of dislocation cores and grain boundaries along a normalized diffusion distance where $\delta_{\mathrm{gb}}$ is the effective width of the grain boundary.

We now consider the combined diffusion along the actual dislocation cores $\left(D_{\text {core }}\right)$ and along the adjoining grain boundary $\left(D_{\mathrm{gb}}\right)$, for a normalized diffusion distance, $L . D_{\text {core }}^{\text {eff }}$ is simply the dislocation core diffusivity when grain boundary diffusion for a mean distance, $R$, has been considered. This is again illustrated in Fig. 14b. $\delta_{\mathrm{gb}}$ is the effective width of the grain boundary.

The mass flow along a dislocation core, $q_{\text {core }}$, is simply

$$
q_{\text {core }}=J_{\text {eff }} a_{\text {core }},
$$

as is clear from Fig. $14 \mathrm{a}$, where $a_{\text {core }}$ is the crosssectional area of a dislocation core $\left(=\pi r_{\text {core }}^{2}\right)$. The mass flows in Fig. 14b consist of:

(1) mass flow along the dislocation core, $q_{\text {core }}$

(2) mass flow along the grain boundary region, $q_{\mathrm{gb}}$

Now considering the diffusion along a dislocation core of length, $L$, as driven by the chemical potential difference, $\Delta \mu_{1}$, it is expected that

$$
J_{1}=-\frac{D_{\text {core }}}{k T \Omega} \frac{\Delta \mu_{1}}{L},
$$

which leads to

$$
q_{\text {core }}=J_{1} a_{\text {core }}=\left(-\frac{D_{\text {core }}}{k T \Omega} \frac{\Delta \mu_{1}}{L}\right)\left(\pi r_{\text {core }}^{2}\right) .
$$

Along the grain boundary region, which can be modeled as a donut-shaped disc (Fig. 15) with disc thickness $\delta_{\mathrm{gb}}$, inner diameter $r_{\text {core }}$, and outer diameter $R$, the diffusion can be described as follow

$$
q_{\mathrm{gb}}=J_{2}\left(2 \pi r \delta_{\mathrm{gb}}\right),
$$

or equivalently,

$$
q_{\mathrm{gb}}=2 \pi r \delta_{\mathrm{gb}}\left\{-\left(\frac{D_{\mathrm{gb}}}{k T \Omega}\right) \frac{\mathrm{d} \mu}{\mathrm{d} r}\right\}
$$

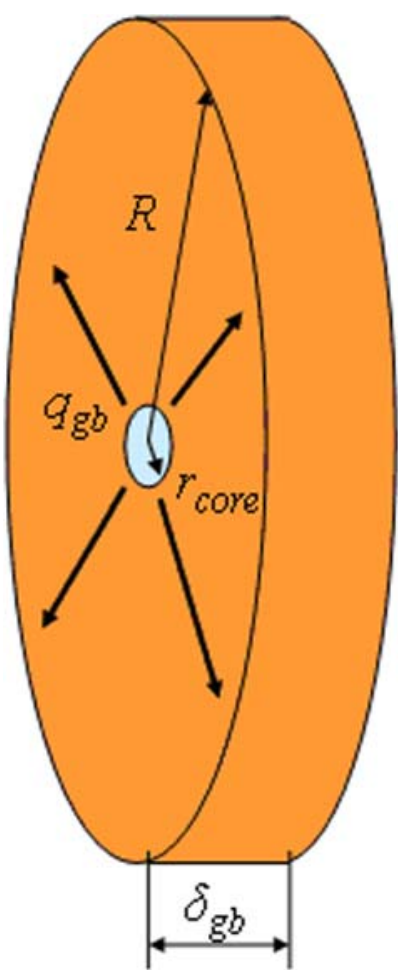

Fig. 15. Illustration of the diffusion along the grain boundary region with indications of the parameters $\left(R, r_{\text {core }}, q_{\mathrm{gb}}\right.$, and $\left.\delta_{\mathrm{gb}}\right)$ as used in the analyses.

where $q_{\mathrm{gb}}$ is the mass flow along the grain boundary, and $J_{2}$ is the grain boundary mass flow per area, while the variables $\mu$ and $r$ are the chemical potential and radius/distance of diffusion from the center of the disc, respectively. However $q_{\mathrm{gb}}$ is not a function of $r(\neq f(r))$, and thus should be a constant. Consequently, by rearranging Eq. 12, we find

$$
-q_{\mathrm{gb}} \frac{k T \Omega}{2 \pi \delta_{\mathrm{gb}} D_{\mathrm{gb}}}=\frac{\mathrm{d} \mu}{\mathrm{d} r}=\text { const. }
$$

Integrating and further rearranging leads to

$$
\Delta \mu_{2}=-q_{\mathrm{gb}} \frac{k T \Omega}{2 \pi \delta_{\mathrm{gb}} D_{\mathrm{gb}}} \ln \left(\frac{R}{r_{\text {core }}}\right),
$$

where $\Delta \mu_{2}$ is simply the integrated chemical potential difference for diffusion along the grain boundary region from $r=r_{\text {core }}$ to $r=R$.

In the combined diffusion along the actual dislocation core and along the grain boundary region (Fig. 14b), mass conservation requires

$$
q_{\text {core }}=q_{\mathrm{gb}},
$$

which after substituting Eqs. 10 and 14 would lead to

$$
-\pi r_{\text {core }}^{2} \frac{D_{\text {core }}}{k T \Omega} \frac{\Delta \mu_{1}}{L}=-\Delta \mu_{2} \frac{2 \mu \delta_{\mathrm{gb}} D_{\mathrm{gb}}}{k T \Omega \ln \left(R / r_{\text {core }}\right)} .
$$


Obviously, the combined chemical potential, $\Delta \mu$, can be described as follows

$$
\Delta \mu=\Delta \mu_{1}+\Delta \mu_{2}
$$

Combining Eqs. 16 and 17, we find

$$
\Delta \mu_{1}=\left\{\frac{2 \delta_{\mathrm{gb}} D_{\mathrm{gb}} L}{2 \delta_{\mathrm{gb}} D_{\mathrm{gb}} L+r_{\text {core }}^{2} D_{\text {core }} \ln \left(R / r_{\text {core }}\right)}\right\} \Delta \mu .
$$

To derive the effective diffusivity of the core diffusion, $D_{\text {core }}^{\text {eff }}$, we rewrite Eq. 10 as

$$
q_{\text {core }}=-\pi r_{\text {core }}^{2} \frac{D_{\text {core }}}{k T \Omega} \frac{\Delta \mu_{1}}{L},
$$

and by substituting Eq. 18, we find

$$
\begin{aligned}
q_{\text {core }}= & -\pi r_{\text {core }}^{2} \frac{D_{\text {core }}}{k T \Omega} \\
& \times\left\{\frac{2 \delta_{\mathrm{gb}} D_{\mathrm{gb}} L}{2 \delta_{\mathrm{gb}} D_{\mathrm{gb}} L+r_{\text {core }}^{2} D_{\text {core }} \ln \left(R / r_{\text {core }}\right)}\right\} \frac{\Delta \mu}{L},
\end{aligned}
$$

which suggests that

$$
D_{\text {core }}^{\text {eff }}=D_{\text {core }}\left\{\frac{2 \delta_{\text {gb }} D_{\text {gb }} L}{2 \delta_{\mathrm{gb}} D_{\text {gb }} L+r_{\text {core }}^{2} D_{\text {core }} \ln \left(R / r_{\text {core }}\right)}\right\} \text {. }
$$

\section{REFERENCES}

1. L.L. Vanasupa, Y.C. Joo, P.R. Besser, and S. Pramanick, J. Appl. Phys. 85, 2583 (1999). doi:10.1063/1.369624.

2. B.C. Valek, J.C. Bravman, N. Tamura, A.A. MacDowell, R. Celestre, H. Padmore, R. Spolenak, W.L. Brown, B.W. Batterman, and J.R. Patel, Appl. Phys. Lett. 81, 4168 (2002). doi:10.1063/1.1525880

3. B.C. Valek, N. Tamura, R. Spolenak, W.A. Caldwell, A. MacDowell, R.S. Celestre, H.A. Padmore, J.C. Bravman, B.W. Batterman, W.D. Nix, and J.R. Patel, J. Appl. Phys. 94, 3757 (2003). doi:10.1063/1.1600843.

4. A.S. Budiman, N. Tamura, B.C. Valek, K. Gadre, J. Maiz, R. Spolenak, W.D. Nix, and J.R. Patel, Appl. Phys. Lett. 88, 233515 (2006). doi:10.1063/1.2210451.

5. A.S. Budiman, N. Tamura, B.C. Valek, K. Gadre, J. Maiz, R. Spolenak, W.A. Caldwell, W.D. Nix, and J.R. Patel, Mater. Res. Soc. Symp. Proc. 812, 345 (2004).
6. A.S. Budiman, N. Tamura, B.C. Valek, K. Gadre, J. Maiz, R. Spolenak, J.R. Patel, and W.D. Nix, Mater. Res. Soc. Symp. Proc. 914, 295 (2006).

7. N. Tamura, A.A. MacDowell, R. Spolenak, B.C. Valek, J.C. Bravman, W.L. Brown, R.S. Celestre, H.A. Padmore, B.W. Batterman, and J.R. Patel, J. Synchrotron Radiat. 10, 137 (2003). doi:10.1107/S0909049502021362.

8. R.W. Cahn, J. Inst. Met. 86, 121 (1949).

9. J.F. Nye, Acta Metall. 1, 153 (1953). doi:10.1016/0001-6160 (53)90054-6.

10. P. Besser, E. Zschech, W. Blum, D. Winter, R. Ortega, S. Rose, M. Herrick, M. Gall, S. Thrasher, M. Tiner, B. Baker, G. Braeckelmann, L. Zhao, C. Simpson, C. Capasso, H. Kawasaki, and E. Weitzman, J. Electron. Mater. 30, 320 (2001). doi:10.1007/s11664-001-0038-7.

11. J.E. Sanchez Jr., and P.R. Besser, Proceedings of the International Interconnect Technology Conference (IEEE, Piscataway, NY, 1998), p. 233.

12. J.M. Paik, K.C. Park, and Y.C. Joo, J. Electron. Mater. 33, 48 (2004). doi:10.1007/s11664-004-0293-5.

13. S.P. Baker, Y.C. Joo, M.P. Knaub, and E. Artz, Acta Mater. 48, 2199 (2000).

14. H.J. Frost and M.F. Ashby, Deformation-Mechanism Maps: The Plasticity and Creep of Metals and Ceramics (Oxford: Pergamon, 1982), p. 21.

15. D. Gan, P.S. Ho, Y. Pang, R. Huang, J. Leu, J. Maiz, and T. Scherban, J. Mater. Res. 21, 1512 (2006). doi:10.1557/jmr. 2006.0196.

16. B. Cai, Q.P. Kong, L. Lu, and K. Lu, Scr. Mater. 41, 755 (1999). doi:10.1016/S1359-6462(99)00213-4.

17. W. Dickenscheid, R. Birringer, H. Gleiter, O. Kanert, B. Michel, and B. Gunther, Solid State Commun. 79, 683 (1991). doi:10.1016/0038-1098(91)90613-Z.

18. Z. Suo, Acta Metall. Mater. 42, 3581 (1994). doi:10.1016/ 0956-7151(94)90424-3.

19. A.S. Oates, J. Appl. Phys. 79, 163 (1996). doi:10.1063/ 1.360925 .

20. J.R. Black, Sixth Annual IEEE Intl. Rel. Phys. Symp. Proc., 1967, p. 148.

21. M. Shatzkes and J.R. Llyod, J. Appl. Phys. 59, 3890 (1986). doi:10.1063/1.336731.

22. K.A. Danso and L. Tullos, Microelectron. Reliab. 21, 513 (1981). doi:10.1016/0026-2714(81)90242-0.

23. H.A. Schafft, T.C. Grant, A.N. Saxena, and C.Y. Kao, $23 r d$ Annual IEEE Intl. Rel. Phys. Symp. Proc., 1985, p. 93.

24. R. Kirchheim and U. Kaeber, J. Appl. Phys. 70, 172 (1991). doi:10.1063/1.350305.

25. A.S. Budiman, C.S. Hau-Riege, P.R. Besser, A. Marathe, Y.-C. Joo, N. Tamura, J.R. Patel, and W.D. Nix, 45th Annual IEEE Intl. Rel. Phys. Symp. Proc., 2007, pp. 122-127.

26. A.S. Budiman, C.S. Hau-Riege, P.R. Besser, A. Marathe, Y.-C. Joo, N. Tamura, J.R. Patel, and W.D. Nix, 9th Intl. Workshop on Stress-induced Phenomena in Metallization AIP Proc., vol. 945, 2007, p. 56.

27. E. Zschech, M.A. Meyer, and E. Langer, Mater. Res. Soc. Proc. 812, F.7.5.1 (2004). 